\title{
Water maser follow-up of the Methanol Multi-Beam Survey.
}

\author{
Anita Titmarsh ${ }^{1,2}$, Simon Ellingsen ${ }^{1}$, Shari Breen ${ }^{2}$, James Caswell $^{2}$ \\ and Maxim Voronkov ${ }^{2}$ \\ ${ }^{1}$ School of Mathematics and Physics, University of Tasmania, \\ Private Bag 37, Hobart, Tasmania 7001, Australia \\ email: Anita.Titmarsh@utas.edu.au \\ ${ }^{2}$ CSIRO Astronomy and Space Science, \\ PO Box 76, Epping, NSW 1710
}

\begin{abstract}
The Australia Telescope Compact Array has been used to observe all the $6036.7 \mathrm{GHz}$ methanol masers detected in the Methanol Multi-Beam survey between $l=310^{\circ}-20^{\circ}$. To date we have measured positions with arcsecond accuracy for all the observations in the $l=6^{\circ}-20^{\circ}$.
\end{abstract}

Keywords. masers, stars: formation, radio lines: ISM.

\section{Introduction}

Common masers such as the $22 \mathrm{GHz}$ water and $6.7 \mathrm{GHz}$ methanol masers are important tools for studying the formation of high-mass stars. They are common, intense, and being observable at radio frequencies they allow us to probe deep into the heart of the dusty molecular envelope where high-mass stars are forming. Water masers are the most common maser species known, tracing shocked gas, outflows and dense circumstellar shells around evolved stars. They are found at sites of both low and high-mass star formation. In contrast, some other species (e.g. $6.7 \mathrm{GHz}$ methanol masers) are observed exclusively at sites of high-mass star formation (Minier et al., 2003; Xu et al., 2008). The presence and/or absence of various maser transitions are thought to trace different evolutionary stages in their formation (Breen et al., 2010); however, further work is required to quantify the timescale over which the very important water maser transition occurs.

Previous targeted observations of water masers have been carried out with single dish telescopes or have searched 'special' sources. For example Szymczak et al. (2005) observed $796.7 \mathrm{GHz}$ methanol masers with the Effelsberg telescope achieving a spatial resolution of $\sim 40$ arcseconds. Because water masers are very common around regions of star formation, a positional accuracy of at least a few arcseconds is required to reliably identify if the water and methanol masers are coincident with the same object. Beuther et al. (2002) observed a sample of young stellar objects with the VLA (achieving the necessary spatial resolution) and found a detection rate of $62 \%$. However, their sample of $6.7 \mathrm{GHz}$ methanol masers were chosen using IRAS-based selection criteria, which are known to miss a substantial fraction of $6.7 \mathrm{GHz}$ methanol masers (Ellingsen et al., 1996).

The Methanol Multi-Beam (MMB) survey is an unbiased survey of the Galactic Plane $0^{\circ}<l<360^{\circ}$ and $b \pm 2^{\circ}$ for the $6.7 \mathrm{GHz}$ methanol $6.035 \mathrm{GHz}$ excited $\mathrm{OH}$. The southern portion of the MMB survey was completed in March 2009 (Green et al., 2009) and $l=186^{\circ}-20^{\circ}$ have been published (Caswell et al., 2010, 2011; Green et al., 2010, 2012). The Parkes $64 \mathrm{~m}$ dish performed the initial search of the southern Galactic Plane and 
accurate positions for the sites of maser emission detected here were then followed up with the ATCA.

Here we report preliminary results of follow-up $22 \mathrm{GHz}$ water maser observations towards the MMB detections. Although these observations are targeted (rather than an unbiased search), we will be able to combine the results of these sensitive, high resolution observations, with the less sensitive, but statistically complete HOPS survey (Walsh et al., 2011), to properly quantify the water maser transition with the maser-based evolutionary scheme.

\section{Water maser observations}

The observations were made between November 2010 and August 2011 with the ATCA in various antenna configurations. The observations on the 2 nd and $3 \mathrm{rd}$ of November 2010 were carried out in the H214 array configuration, and the observations on the 9th and 10th of August 2011 were in the H168 configuration. These hybrid array configurations have both East - West and North - South baselines and are better for observations of equatorial sources, although at the cost of a larger synthesised beam. The primary beam of the compact array at $22 \mathrm{GHz}$ is 2.1 arcminutes and the synthesised beams of the $\mathrm{H} 214$ and H168 configurations are $\sim 9.6$ and $\sim 12.4$ arcminutes respectively. It is important to realise that the astrometric accuracy for an connected-element interferometer such as the ATCA depends upon both the size of the synthesised beam, and the quality of the phase calibration. For observations at $22 \mathrm{GHz}$ observations with longer baselines (and hence smaller synthesised beams), will not necessarily lead to better astrometric accuracy if the quality of the phase calibration for those longer baselines is poor. In good observing conditions the absolute astrometric accuracy for the ATCA is around 0.4 arcseconds (set by the astrometric accuracy of the phase calibrators and the degree to which they are point sources for the array configuration and the frequency of observation). For our observations the astrometric accuracy is in the range $0.4-2.0$ arcseconds, as some of the observations were made in relatively poor weather. This accuracy is comparable to the maximum angular extent observed for water maser clusters by Breen et al. (2011).

Targeted observations of each of the $6.7 \mathrm{GHz}$ methanol masers were performed with at least four observations of 1.5 minutes duration spread over an hour angle range of 6 hours to ensure sufficient $\mathrm{u}-\mathrm{v}$ coverage for imaging. The sensitivity in an individual spectral channel for these observations ranged from $\sim 40 \mathrm{mJy}$ in good weather conditions to $\sim 80 \mathrm{mJy}$ in poor weather. The Compact Array Broadband Backend (CABB) with two zoom bands of $64 \mathrm{MHz}$ and $32 \mathrm{kHz}$ resolution were used to observe the $22 \mathrm{GHz}$ water maser transition in the first zoom band and the the ammonia $(1,1)$ and $(2,2)$ transitions in the second zoom band. The velocity coverage in the zoom bands was $>800 \mathrm{kms}^{-1}$ with velocity resolution of $0.42 \mathrm{kms}^{-1} .2 \times 2 \mathrm{GHz}$ continuum bands were also available for the August 2011 observations.

\section{Results for $l=6^{\circ}-20^{\circ}$}

Here we report the water maser observations for the Galactic longitude range $l=$ $6^{\circ}-20^{\circ}$. Approximately $40 \%$ of the $6.7 \mathrm{GHz}$ methanol masers in this region have an associated water maser. The criteria we used to determine association between the water and methanol masers was if they have an angular separation of less than two arcseconds. This criterion was used for consistency with previous high resolution, large surveys of water masers (eg. Breen et al. 2010). Our detection rate is lower than other targeted surveys of water masers such as Szymczak et al. (2005) who used the Effelsberg telescope 

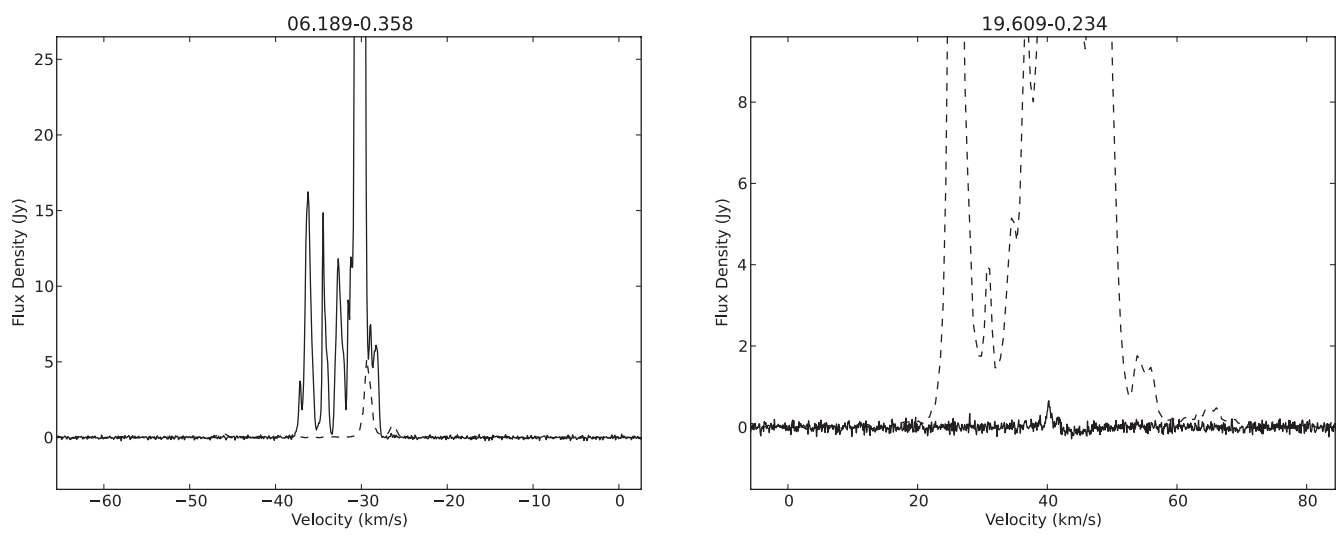

Figure 1. Spectra of associated $22 \mathrm{GHz}$ water masers taken with the ATCA (shown with dashed lines) and 6.7 GHz methanol masers from the MMB survey (shown with solid lines).

(half power beam width of 40 arcseconds at $22 \mathrm{GHz}$ ) and found $52 \%$ of the $6.7 \mathrm{GHz}$ methanol masers had an associated water maser. Since our survey is also more sensitive we would expect to have a higher detection rate. However, if we relax the association criteria to include masers within 10 arcseconds then our detection rate increases to $79 \%$.

Comparing the $6.7 \mathrm{GHz}$ methanol masers with and without associated water masers we found that the average peak flux densities of the methanol masers were higher in those without water masers (average of $92 \mathrm{Jy}$ for sources without water and $36 \mathrm{Jy}$ for those with water). However, this result is strongly affected by the famous and unusual source G09.621+0.196 which had a peak flux density of 5196 Jy at the time of the MMB observations, it has also been observed to have periodic flares (eg. Goedhart et al. 2003). Disregarding this source changes the result so methanol masers with associated water masers have greater average peak flux densities (24 Jy for sources without water and $49 \mathrm{Jy}$ with water) which would be expected if water masers occurred at a later stage during star formation.

Breen et al. (2010) showed that the luminosity of the $6.7 \mathrm{GHz}$ methanol masers increases as they evolve. Comparing the $6.7 \mathrm{GHz}$ methanol maser peak flux densities with their associated water maser peak flux densities (Fig. 2) suggests a correlation between the flux densities and is consistent with the scenario that water masers also increase in intensity as they evolve. This is a loose correlation, and the Pearson correlation coefficient is 0.37 . However, this is strongly affected by one very strong source and without it the correlation coefficient reduces to 0.26 . The sources observed in this survey with the most extreme differences between water and methanol peak flux densities are shown in Figure 1. Source G06.189-0.358 has a strong methanol maser (221.6 Jy) and weak water maser (5.2 Jy) while G19.609-0.234 is the reverse with strong water (33.4 Jy) and weak methanol (1 Jy).

Water masers are well known for sometimes exhibiting high velocity emission, offset from the systemic emission of the region by $100 \mathrm{kms}^{-1}$ or more. Figure 3 shows a comparison of the velocities of the peak emission of the two maser species. In most masers the water and methanol masers have peak velocities within a few $\mathrm{kms}^{-1}$ of each other. This suggests that for water masers associated with $6.7 \mathrm{GHz}$ methanol masers the peak emission is in most cases close to the systemic velocity of the region and any high-velocity emission is generally weaker. One notable exception is the methanol maser source G18.999-0.239. The $6.7 \mathrm{GHz}$ methanol emission peaks at $69.4 \mathrm{kms}^{-1}$ whereas the 


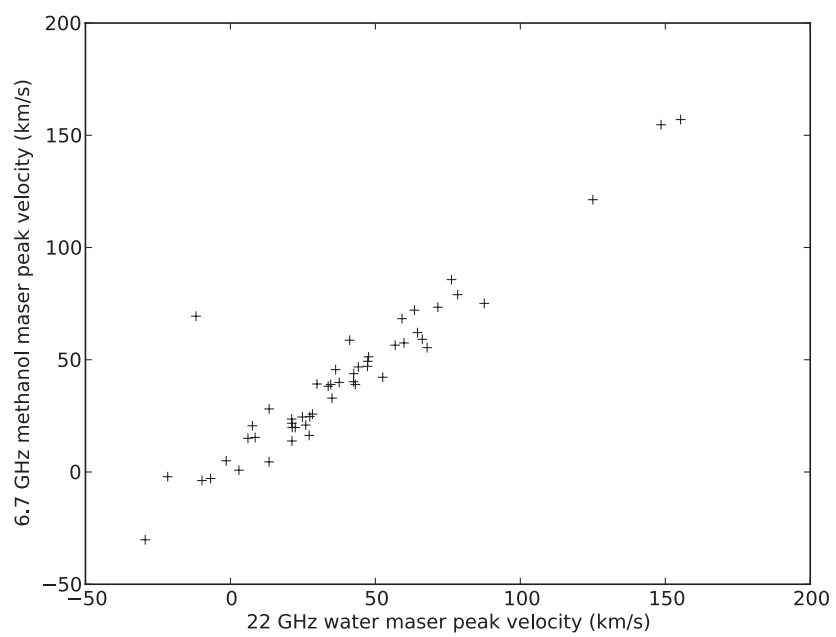

Figure 2. $22 \mathrm{GHz}$ water maser peak velocity $\left(\mathrm{kms}^{-1}\right)$ vs. $6.7 \mathrm{GHz}$ methanol maser peak velocity $\left(\mathrm{kms}^{-1}\right)$.

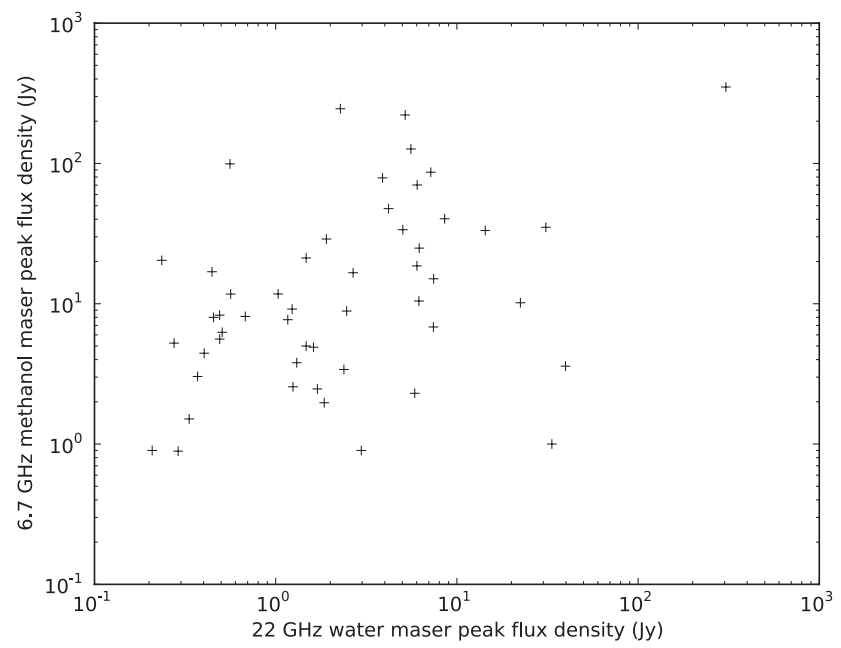

Figure 3. $22 \mathrm{GHz}$ water maser peak flux density (Jy) vs. $6.7 \mathrm{GHz}$ methanol maser peak flux density (Jy).

water maser emission peaks at $-11.8 \mathrm{kms}^{-1}$. They are only separated by 1.4 arcseconds and neither maser species shows any emission around the others peak velocity.

\section{References}

Breen, S. L., Caswell, J. L., Ellingsen, S. P., \& Phillips, C. J. 2010, MNRAS, 406, 1487

Breen, S. L. \& Ellingsen, S. P. 2011, MNRAS, 416, 178

Beuther et al. 2002, A\&A, 390, 289

Caswell et al. 2010, MNRAS, 404, 1029

Caswell et al. 2011, MNRAS, 417, 1964

Ellingsen et al. 1996, MNRAS, 280, 378

Goedhart S., Gaylard M. J., \& van der Walt D. J. 2003, MNRAS, 339, L33

Green et al. 2009, MNRAS, 392, 783 
Green et al. 2010, MNRAS, 409, 913

Green et al. 2012, MNRAS, 420, 3108

Minier, V., Ellingsen, S. P., Norris, R. P., \& Booth, R. S. 2003, A\&AA, 403, 1095

Szymczak, M., Pillai, T., \& Menten, K. M. 2005, A\& $A$, 434, 613

Walsh et al. 2011, MNRAS, 416,1764

$\mathrm{Xu}$ et al. 2008, A\&BA, 485, 729 